\title{
Kinematic analysis of motor strategies in frail aged adults during the Timed Up and Go: how to spot the motor frailty?
}

This article was published in the following Dove Press journal:

Clinical Interventions in Aging

26 February 2015

Number of times this article has been viewed

\author{
Asma Hassani' \\ Alexandre Kubicki ${ }^{2,3}$ \\ Vincent Brost ${ }^{1}$ \\ France Mourey ${ }^{2,4}$ \\ Fan Yang'
}

\begin{abstract}
'Laboratoire LE2I CNRS 6306,
Université de Bourgogne, Dijon,

France; ${ }^{2}$ Institut National de la Santé et de la Recherche Médicale (INSERM), Cognition Action et Plasticité SensoriMotrice, Campus Universitaire, Université de Bourgogne, Dijon, France; ${ }^{3}$ Centre Hospitalier Universitaire de Dijon, Hôpital de Champmaillot, Dijon, France; ${ }^{4}$ Faculté de Médecine, Université de Bourgogne, Dijon, France
\end{abstract}

Objective: The purpose of this work was to analyze and compare the movement kinematics of sit-to-stand (STS) and back-to-sit (BTS) transfers between frail aged adults and young subjects, as well as to determine the relationship between kinematic changes and functional capacities. Methods: We analyzed the Timed Up and Go (TUG) movements by using a 3D movement analysis system for real-time balance assessment in frail elderly. Ten frail aged adults (frail group $[\mathrm{FG}]$ ) and ten young subjects (young group [YG]) performed the TUG. Seven spatiotemporal parameters were extracted and compared between the two groups. Moreover, these parameters were plotted with TUG test duration.

Results: The experiments revealed that there were significant differences between FG and YG in trunk angle during both STS and BTS, and in TUG duration. The trunk angle of the young subjects was more than two times higher than that of the FG. As expected, the TUG duration was higher in the FG than in YG. Trunk angles during both transfers were the most different parameters between the groups. However, the BTS trunk angle and STS ratio were more linked to functional capacities.

Conclusion: There was a relationship between kinematic changes, representing the motor planning strategies, and physical frailty in these aged adults. These changes should be taken into account in clinical practice.

Keywords: aging, Timed Up and Go test, kinematics, frailty effects

\section{Introduction}

The balance function consists of maintaining equilibrium during both stationary conditions and mobility. It is a complex function resulting from the interaction of several physiological systems, which changes with age. The loss of physiological reserves caused by aging and chronic diseases leads to a frail state. Frailty is a significant geriatric syndrome characterized by age-associated severely reduced strength, ${ }^{1}$ mobility, balance, and endurance. ${ }^{2}$ This decrease in physiological reserves may result in falls, disability, hospitalization, or even death..$^{2-4}$ Sarcopenia, a common syndrome in older adults, is considered to play a crucial role in the frailty process. It is characterized by a progressive loss of muscle mass and muscle power. ${ }^{5}$ Its causes are multifactorial and can include hormonal factors and nutritional deficiencies. ${ }^{6}$ Sarcopenia can lead to poor outcomes, including fatigue, falls, physical disabilities, and functional decline, ${ }^{7,8}$ exacerbating a vicious circle involving physical inactivity and deconditioning and decreased energy expenditure. ${ }^{9}$

However, the decrease in physical capacities is not only a consequence of muscle weakness. It could be caused by planning impairment and movement disorganization. Saimpont et al investigated age-related changes in action simulation/action planning.
Correspondence: Alexandre Kubick INSERM UI093, Cognition, Action, et Plasticité Sensori-Motrice, BP 27877, Campus Universitaire, Université de Bourgogne, Dijon, France

Tel +33380396767

Fax +33380396702

Email alexandre.kubicki@u-bourgogne.fr 
Their finding supported the hypothesis of a decline in action planning with aging. ${ }^{10}$ Indeed, the results showed that older adults were impaired in their ability to mentally simulate the rising from the floor sequence, at least through an imagesequencing task: they made significantly more sequencing errors than did their younger counterparts. The work of Allain et al showed that aged patients with cognitive disorders have problems with mentally developing logical strategies and executing complex predetermined plans, which are partially related to behavioral changes. ${ }^{11}$

From a functional point of view, these problems of planning abilities may affect the quality of the sit-to-stand (STS) and back-to-sit (BTS) transfers. In the study by Mourey et $\mathrm{al}^{12}$ the effects of aging on kinematic features of body motion during STS and BTS were explored. Seven healthy young subjects and five healthy older people (mean age $73.2 \pm 5.5$ years) participated in this study, and were compared. Duration and trunk angle for BTS and STS were calculated. During these movements, the trunk control allows subjects to adjust weight shifts, and to perform selective movements in order to maintain the center of mass within the base of support during dynamic postural adjustments. ${ }^{13}$ Considering the STS, for instance, the trunk flexion allows the subject to shift his center of mass toward the future basis of support. Indeed, this trunk movement corresponds to a postural anticipation needed to perform the transfer, and is allowed by the motor planning process. ${ }^{14}$ The results showed that trunk angles were not impaired in these normal aged adults, neither in STS nor in BTS. However, there was an age-related deceleration when performing both transfers. In addition, the healthy older people had more difficulties with curbing the movements accelerated by the force of gravity during the BTS. This contributes to an increase in the time required to achieve this task. These planning problems seem greater in older adults with mild-to-moderate Alzheimer's disease (AD): the study by Manckoundia et al showed that higher level motor processes of whole body motions are affected by $\mathrm{AD}$, while lower level motor features remain intact. ${ }^{15}$ The work involved a comparison of the kinematics of STS and BTS motions between six healthy older adults (mean age $79 \pm 3.5$ years) and six older adults with AD (mean age $81 \pm 3.5$ years). The results showed that the duration of STS and BTS was shorter in the AD group than in the healthy older people group. Moreover, the kinematic analysis revealed that for both STS and BTS, AD subjects performed the task with significantly smaller shoulder displacements than healthy subjects, ie, AD subjects tilted the trunk less than healthy subjects. This is due to the fact that during both STS and BTS, AD subjects reduced their motion in the horizontal plane, especially during STS. Thus, the authors concluded that the AD subjects did not have intact motor planning processes. The implication of the outcomes of the AD group is interesting because various studies have reported that physical frailty is significantly linked to the incidence of dementia. ${ }^{16,17}$ In addition, cognitive impairment is considered to be a component of frailty. ${ }^{18}$

However, there is a lack of literature about the STS and BTS transfers in the frail population. The studies cited above showed light impairment in normal aged adults, mainly in terms of their temporal characteristics, and a more severe impairment in AD patients. Thus, we investigated the planning impairment in the frail older adults both at the level of their temporal characteristics and the changes in their motor strategies. Finally, we will try to link these motor organization deficiencies to functional capacity using the Timed Up and Go (TUG) test, ${ }^{19}$ a clinical test that has been extensively used to assess functional balance and mobility mainly in frail older people. ${ }^{20}$ The TUG consists of standing up from a chair, walking a distance of $3 \mathrm{~m}$, turning, walking back to the chair, and sitting down again. The time taken to complete the test is usually used as the main outcome of analysis and allows, according to some authors, the prediction of the risk of falling. ${ }^{21,22}$ A score of 13.5 seconds is considered as the upper limit of normal mobility, with a sensitivity and a specificity of $0.8 .^{22}$ In this way, we will be able to measure the influence of frailty effects on motor planning during STS and BTS, and to determine the most functional-related kinematic parameter.

\section{Methods \\ Subjects and motor task}

Data were obtained from ten healthy young subjects (young group [YG], two females and eight males) aged 21-30 years (mean age 26.6 \pm 2.95 years) and ten frail aged adults (frail group [FG], six females and four males) aged 83-95 years (mean age 87.9 \pm 4.2 years). FG was composed of patients who were following a functional rehabilitation program managed by only one physiotherapist. These patients were suffering from different pathologies, involving loss of functional independence such as chronic obstructive pulmonary disease (COPD) $(\times 2)$, shoulder, hip $(\times 2)$, and thoracolumbar $(\times 3)$ deficiency (see Table 1 for a description of these patients). They were included in this group according to the Fried criteria of the frailty syndrome (unintentional weight loss, self-reported exhaustion, weakness, slow walking speed, and low physical activity). ${ }^{2}$ The elderly subjects were selected according to 
Table I FG subjects

\begin{tabular}{llll}
\hline Patient & Age (years) & Height $(\mathbf{m})$ & Health problems \\
\hline PI & 94 & 1.55 & COPD, heart insufficiency, arthritis \\
P2 & 83 & 1.65 & \\
P3 & 88 & 1.65 & Dietary supplements \\
P4 & 87 & 1.70 & Idiopathic neuropathy \\
P5 & 95 & 1.50 & Early Alzheimer's disease and arthritis \\
P6 & 83 & 1.65 & \\
P7 & 85 & 1.60 & Ulcerative colitis and neurological problems (sensory deep) at the knee \\
P8 & 87 & 1.60 & COPD treated with bronchial dilators \\
P9 & 86 & 1.60 & Dietary supplements \\
PI0 & 91 & 1.55 &
\end{tabular}

Abbreviations: COPD, chronic obstructive pulmonary disease; FG, frail group.

various inclusion and exclusion criteria. The inclusion criteria required subjects to be aged 65 years or more, capable of performing the STS and BTS, maintaining standing, walking, and making U-turns without human assistance, and had cognitive and behavioral skills adapted to comply with instructions. The exclusion criterion for participation in this study was to have a diagnosis of cognitive impairments as evaluated by the mini-mental state examination (MMSE). All scores of the included patients were $>28 / 30$.

Subjects were seated on a chair with armrests (chair width: $47 \mathrm{~cm}$, seating depth: $40 \mathrm{~cm}$, backrest height: $38 \mathrm{~cm}$, armrest height: $60 \mathrm{~cm}$, and total chair height: $76 \mathrm{~cm}$ ). Their feet were placed flat, and their shanks were flexed $\left(10^{\circ}\right.$ with respect to the vertical axis). The chair was placed at knee height and its backrest allowed the subjects to maintain their trunk in a vertical position. From this initial position, subjects were asked to complete three trials of the TUG test. They were given verbal instructions to rise from the chair, walk $3 \mathrm{~m}$, cross a mark on the floor, turn around, walk back, and sit down.

\section{Recording system and data analysis}

Kinematic trajectories during TUG were recorded by means of a wearable system for real-time balance assessment in the aged subjects. The instrument used a Kinect sensor that allowed us to detect the subjects' TUG movements. This sensor produces accurate results, especially for when tracking shoulder movements (segment lengths and angle estimation). ${ }^{23}$ The sensor can reach a frame rate of 30 images per second at a resolution of $640 \times 480$ with 8 bits per channel. The infrared camera operates at $30 \mathrm{~Hz}$. The field of view for the system is $58^{\circ}$ horizontal, $45^{\circ}$ vertical, $70^{\circ}$ diagonal, and the operational range is between $0.8 \mathrm{~m}$ and $3.5 \mathrm{~m}$. The Kinect does not require any calibration. It was placed at a distance of $2.5 \mathrm{~m}$ from the chair with a tilt angle of $20^{\circ}$. No markers or wearable sensors were attached to the body. The system analyzed the TUG motions and mainly the STS and BTS motions. Seven spatiotemporal parameters were computed from the gathered data. ${ }^{24}$

\section{Trunk angle}

The trunk angle refers to the angle, measured in degrees, between the trunk and the vertical plane passing through the center of mass of the body (see Figure 1).

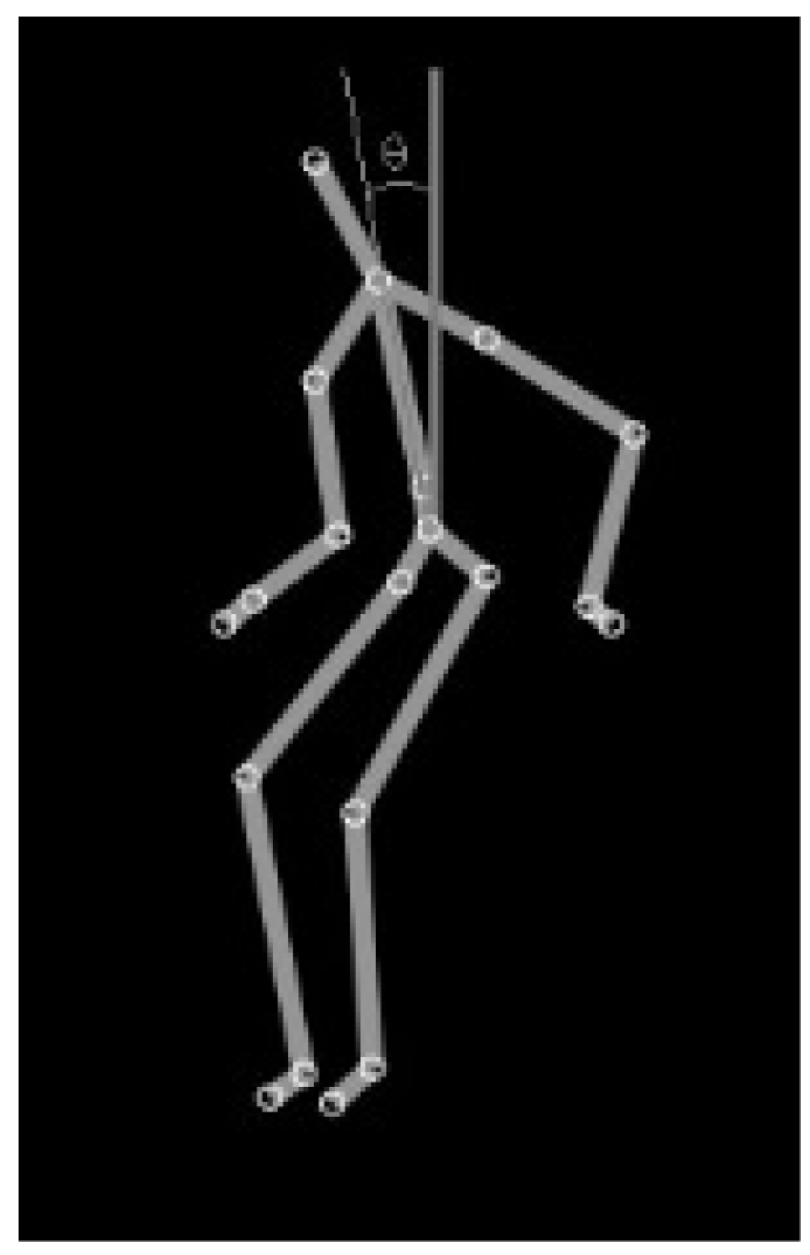

Figure I Trunk angle calculation. 


\section{Ratio}

The ratio is computed as follows:

$$
\text { Ratio }=\frac{D_{v p h}}{D_{h p h}}
$$

where $D_{v p h}$ and $D_{h p h}$ are, respectively, the vertical and the horizontal movement duration.

\section{Movement duration}

The total movement duration of the shoulder, during STS, corresponds to the time interval between the moment when the shoulder depth component (anterior-posterior axis) exceeds $8.5 \%$ of its initial position, corresponding to the lift-off of the buttocks from the seat, and the moment when the head vertical component reaches or exceeds $94 \%$ of the size of the person (ie, when maximum hip, trunk, and knee extension and maximum head flexion velocity are reached). The thresholds were experimentally determined. In BTS, it is defined as the time interval between the moment when the shoulder vertical component drops its peak value, and the moment when the vertical components of the hips reach their minimum values and the trunk angle reaches its limit. The movement duration was measured in seconds.

\section{TUG duration}

The TUG duration, measured in seconds, means the total time taken to perform all TUG tasks. It corresponds to the time interval between the moment when the forward phase starts and the moment when the backward phase ends.

The first three parameters were calculated for each STS and BTS.

\section{Statistical analysis}

We verified the normality of the distributions (Shapiro-Wilk test) and the homogeneity of the variances (Levene's test). A one-way analysis of variance (ANOVA) was performed with the factor 'group' that included young and older adults. To quantify the difference between groups for discriminating parameters (that show group effect), we calculated the effect size by means of Cohen's $d$ parameter. Cohen's $d$ is defined as follows: ${ }^{25}$

$$
d=\frac{\overline{x_{f}}-\overline{x_{y}}}{S_{\text {pooled }}}
$$

where $\bar{x}$ is the mean (average of treatment or comparison conditions) and $S_{\text {pooled }}$ represents the standard deviation of both groups and is given by:

$$
S_{\text {pooled }}=\sqrt{\frac{\left(n_{f}-1\right) s_{f}^{2}+\left(n_{y}-1\right) s_{y}^{2}}{n_{f}+n_{y}}}
$$

where $n$ and $s$ represent the number of subjects and the standard deviation of each group, respectively. $f$ and $y$ correspond to FG and YG, respectively.

To test the relationship between the parameters and the functional capacities, we applied multiple regression analysis in order to determine which of the ratio, trunk angle, and transfer duration parameters, during the STS and BTS, have a greater link to the functional capacities, as assessed by the TUG duration.

All of these statistical analyses were carried out with an alpha level of 0.05 .

\section{Results}

Figure 2 illustrates the results of trunk angle and ratio during STS and BTS for both FG and YG subjects. The average trunk angles during STS for FG and $\mathrm{YG}$ were $16.76^{\circ} \pm 9.44^{\circ}$ and $35.81^{\circ} \pm 9.18^{\circ}$, respectively. During BTS, they were $19.01^{\circ} \pm 15.74^{\circ}$ and $40.33^{\circ} \pm 10.23^{\circ}$ for FG and YG, respectively. Results from the one-way ANOVA showed that there was a statistically significant difference between groups for the STS trunk angle $[F(1,18)=23.12, P<0.001]$ and BTS trunk angle $[F(1,18)=17.56, P<0.001]$. These results are illustrated in Figure 2A and B, respectively. Concerning the ratio, it was $4.93 \pm 5.05$ in the FG and $2.06 \pm 1.527$ in the YG during STS. There was a statistically significant group difference that was noted in the STS ratio $[F(1,18)=6.07, P<0.05]$ (Figure 2C) but not in the BTS ratio (Figure 2D).

Figure $3 \mathrm{~A}$ and $\mathrm{B}$ give the duration of each phase in the two groups. In YG, BTS duration was $1.06 \pm 0.27$ seconds; in FG, it was $1.71 \pm 0.86$ seconds. A statistically significant difference in BTS duration between young and aged adults was shown $[F(1,18)=6.46, P<0.05]$. The TUG scores obtained for both groups are presented in Figure $3 \mathrm{C}$. These results showed a mean score of 18.28 seconds for the FG, with a range from 13.51-35.57 seconds. These results correspond to those of a population of frail individuals. They are potentially fallers, as they are up to the threshold score of 13.5 seconds, as highlighted by ShumwayCook et al concerning the TUG scores' validity. ${ }^{22}$ In YG, the TUG duration was $10.64 \pm 1.37$ seconds. FG subjects spent more time performing the TUG than YG $[F(1,18)=14.02, P<0.01]$.

Table 2 shows the Cohen's $d$ of the five parameters that allow visualizing of the differences between FG and YG. The STS trunk angle, the TUG duration, and the BTS trunk angle were the parameters that had the highest Cohen's $d$.

We applied a multiple regression model. The variance explained by the model was of $R^{2}=0.77$. The statistically significant 
A

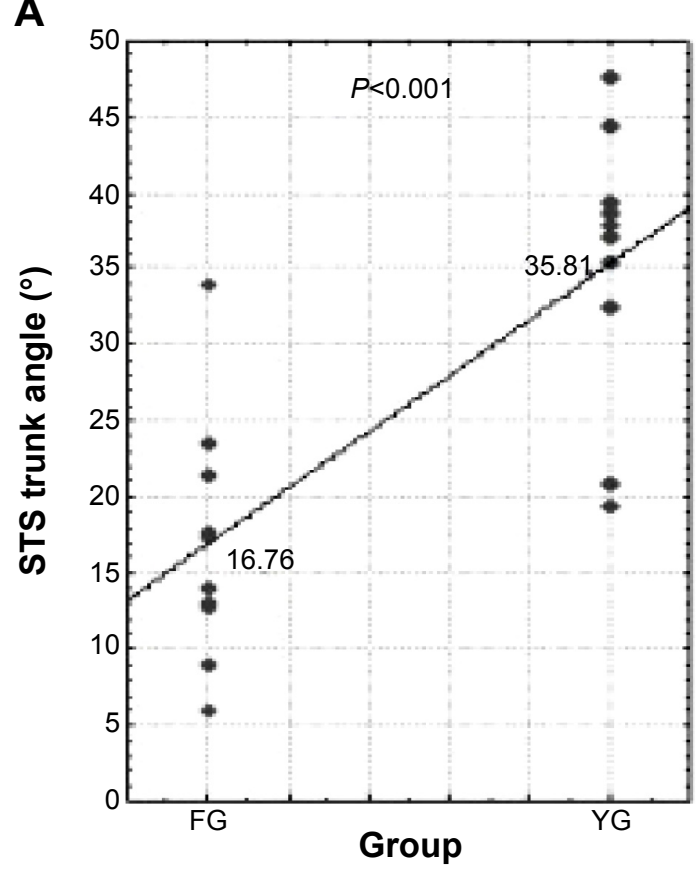

C

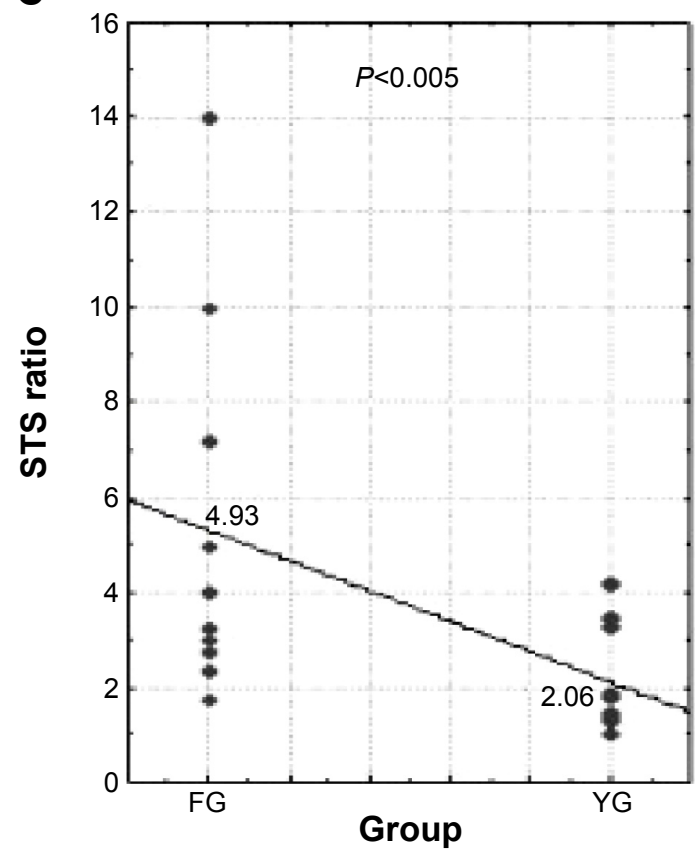

B

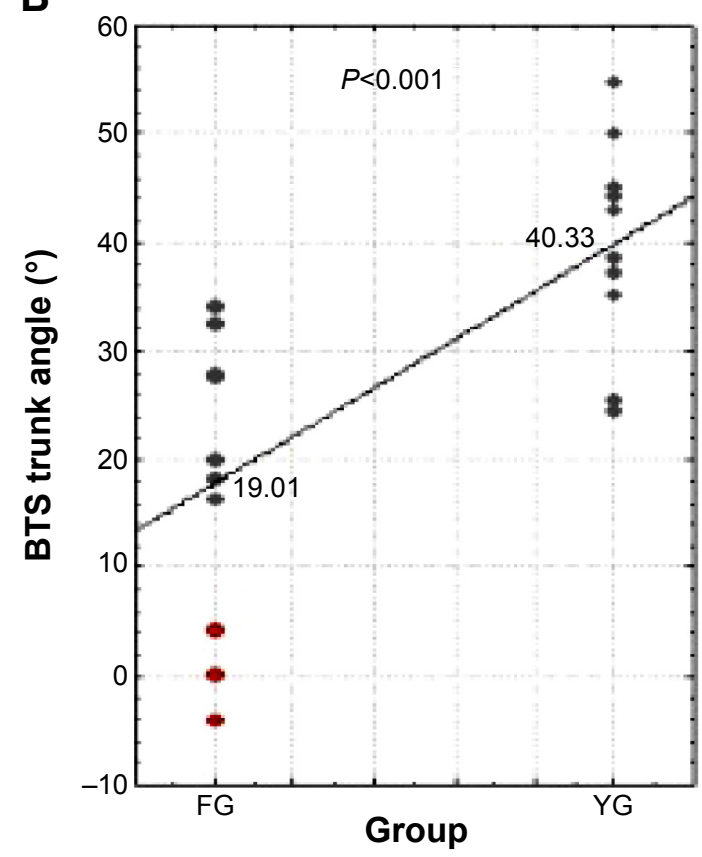

D

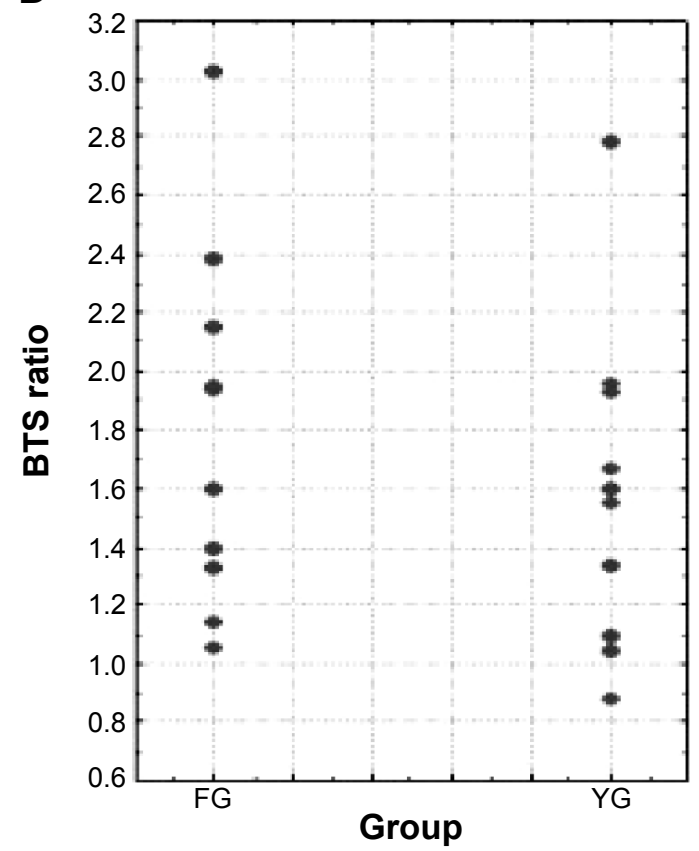

Figure 2 Comparison of trunk angle (A and $\mathbf{B})$ and ratio (C and $\mathbf{D})$ values between ten FG and ten YG subjects during STS and BTS.

Notes: The red circles represent the three subjects with worrying transfers. The solid line passes through the mean values for each group.

Abbreviations: BTS, back-to-sit; FG, frail group; STS, sit-to-stand; YG, young group.

variables are the BTS trunk angle (Beta $=-0.96 ; P=0.021)$ and the STS ratio (Beta $=0.47 ; P=0.035$ ) (Figure $4 \mathrm{~A}$ and $\mathrm{B}$ ).

\section{Discussion}

The main purpose of this study was to compare movement kinematics, during STS and BTS, between FG and YG, and to determine the relationship between these movement characteristics and the functional capacities.
One of the main findings in our study was that there were differences between FG and YG subjects in terms of five kinematic parameters. Among these parameters, the most different were the BTS trunk angle, TUG duration, and the STS trunk angle.

Concerning the TUG duration, the comparisons between the two groups revealed that durations for YG are significantly shorter than for FG. The values were less than 

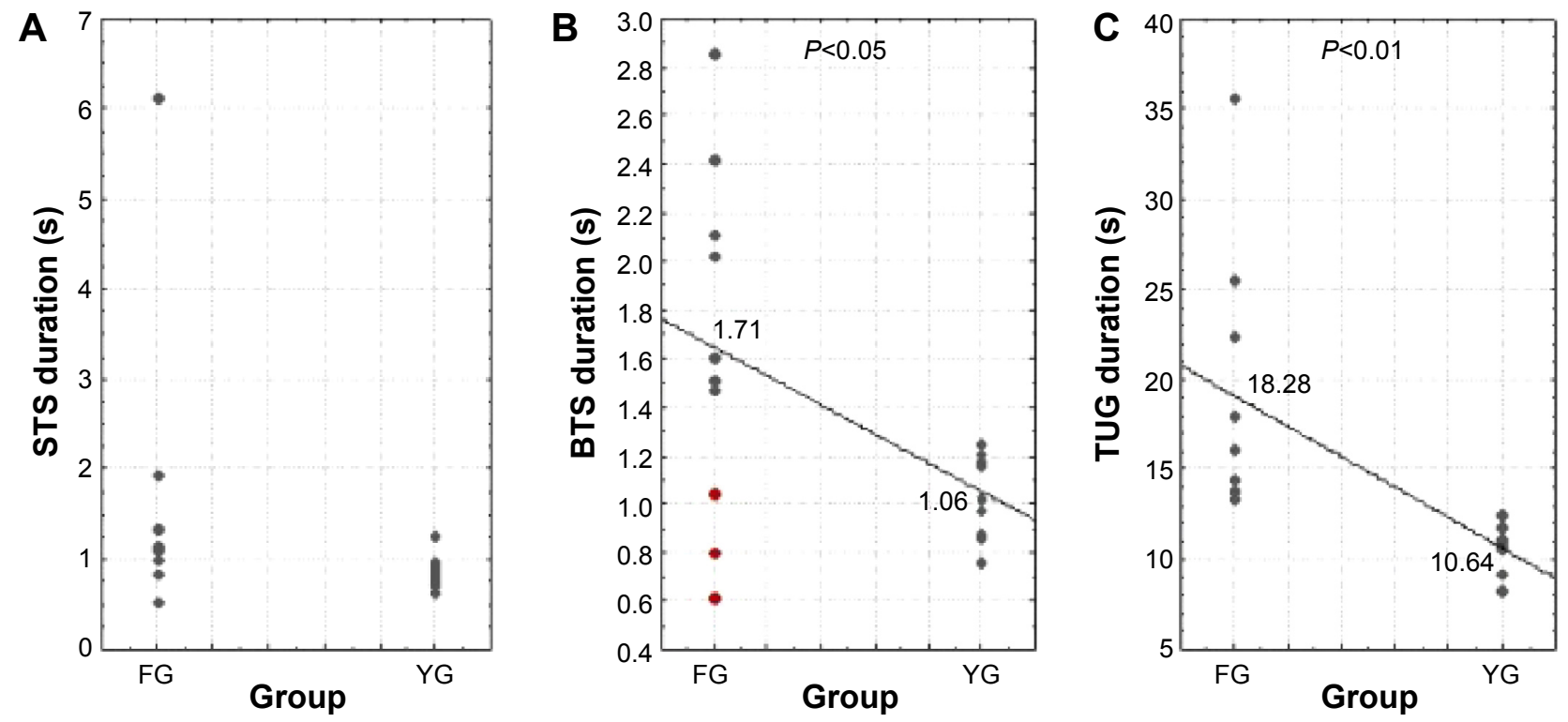

Figure 3 Distribution of the STS (A), BTS (B), and TUG (C) durations for ten FG and ten YG subjects.

Notes: The red circles represent the three subjects with worrying transfers. The solid line passes through the mean for each group.

Abbreviations: BTS, back-to-sit; FG, frail group; STS, sit-to-stand; TUG, Timed Up and Go; YG, young group.

12 seconds in the YG, referring to healthy subjects. In FG, the mean TUG duration was 18.28 seconds, which corresponds to a score of relative frailty.

The mean trunk angle during STS movement was $35.81^{\circ}$ in $\mathrm{YG}$ and $16.76^{\circ}$ in $\mathrm{FG}$. These results are consistent with previous results. In the study by Cignetti et $\mathrm{al}^{26}$ the mean amplitude of trunk flexion during STS was about $36^{\circ}$ in young adults (28.16 \pm 5.75 years). In the study by Mourey et $\mathrm{al}^{12}$ trunk angle displacement during STS was $32^{\circ} \pm 9^{\circ}$ for both healthy young subjects and healthy aged subjects. The frail subjects of our study showed an altered trunk angle: these angles for our young subjects were comparable with those of the normal aged adults from the study by Mourey et $\mathrm{al}^{12}$ and more than twice the angle in our frail group. In the work of Manckoundia et $\mathrm{al}^{15}$ trunk angles were not directly calculated, but $\mathrm{AD}$ patients presented less shoulder displacements on the horizontal phase compared with normal aged subjects. In other words, their trunk flexion was clearly impaired and

Table 2 Cohen's $d$ of the parameters that allow visualization of differences between FG and YG subjects

\begin{tabular}{ll}
\hline Parameters & $\boldsymbol{d}$ \\
\hline STS trunk angle & 2.154 \\
TUG duration & 1.762 \\
BTS trunk angle & 1.692 \\
BTS duration & 1.072 \\
STS ratio & 0.808
\end{tabular}

Abbreviations: BTS, back-to-sit; FG, frail group; STS, sit-to-stand; TUG, Timed Up and Go; YG, young group. the authors concluded that higher level motor processes of whole body motions are affected by AD. The results from our frail aged subjects without cognitive disorders, seem to be relatively close to the motor behavior of the AD subjects assessed in the work by Manckoundia et al. ${ }^{15}$

The durations of these transfers are also interesting. In this study, STS duration was shorter than BTS duration for both frail aged and young subjects, which is in accordance with the work of Manckoundia et $\mathrm{al}^{15}$ and Papaxanthis et al. ${ }^{27}$ STS is an upward movement and is therefore performed against gravity, whereas BTS is a downward movement and is therefore performed with gravity. Thus, this time difference can be attributed to the mechanical effects of gravity ${ }^{15}$ and the braking required against gravity effects that requires fine postural control during the BTS in order to preserve body equilibrium. ${ }^{12}$ It may also be attributed to the absence of visual information during the BTS movement. In our results, frail aged adults executed BTS motions significantly more slowly than young subjects, and these age-related differences appeared much more obviously in BTS than in STS (see effect sizes on Table 2). According to the work of Mourey et $\mathrm{al}^{12}$ with normal aged adults, this behavior adopted by the frail aged adults seems to translate to a cautious motion linked to a difficulty in dealing with the effects of gravity in this transfer. But in addition to this temporal change, the frail subjects also showed some kinematic changes, similar to those of $\mathrm{AD}$ patients, that seem to refer to motion organization deficiencies. This kind of motor planning change 

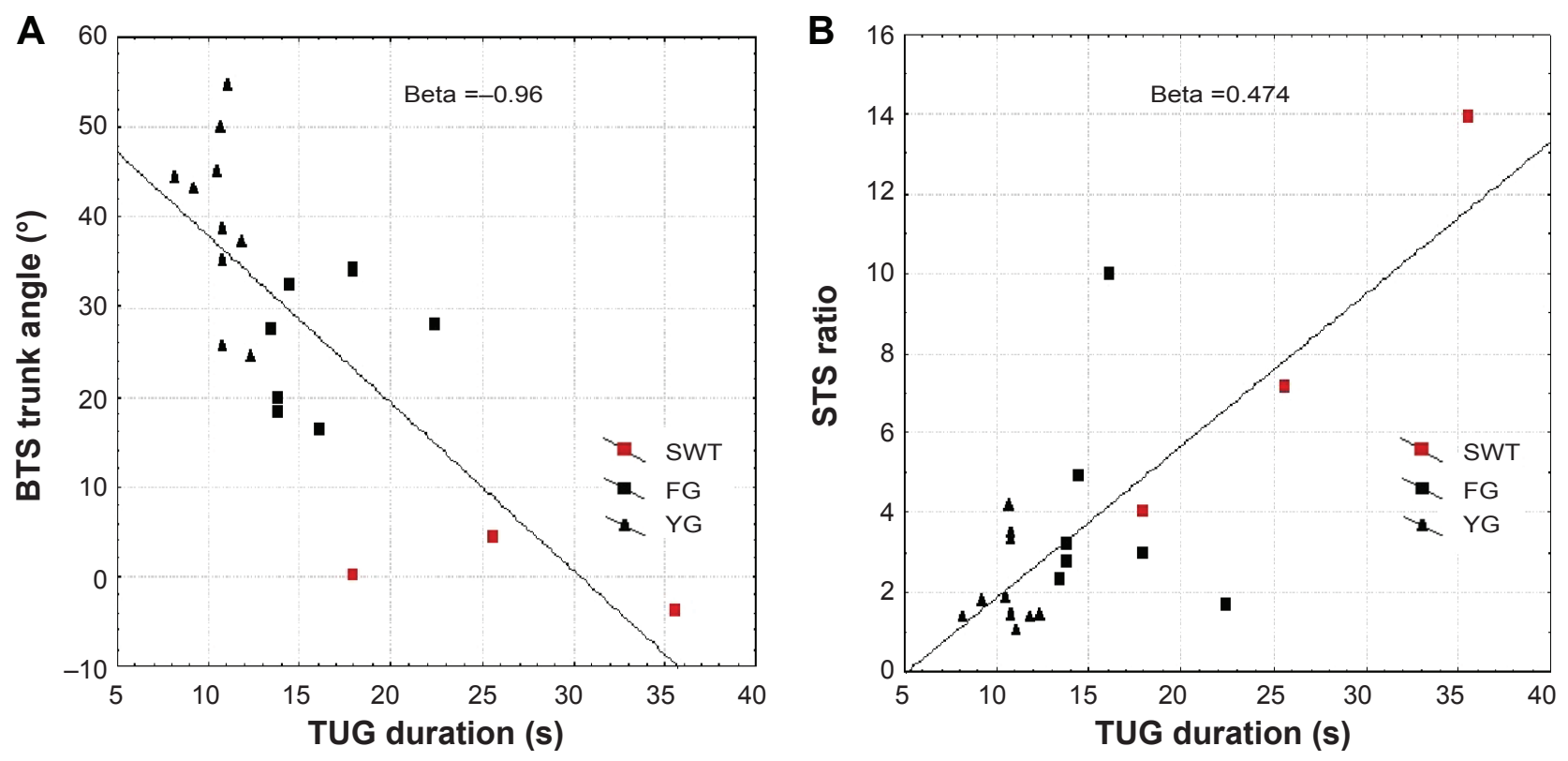

Figure 4 The distribution of the BTS trunk angles (A) and the STS ratio (B) relative to the TUG duration.

Notes: The red circles represent the three subjects with worrying transfers. The solid line denotes the line of best fit.

Abbreviations: BTS, back-to-sit; STS, sit-to-stand; TUG, Timed UP and Go; FG, frail group; YG, young group.

(less trunk flexion) could be interpreted as an impairment of motor prediction (ie, anticipatory postural adjustments), that have been clearly shown to be impaired in older adults, ${ }^{28,29}$ especially in the case of frailty. ${ }^{30}$

However, if the mean BTS duration is higher in frail patients compared to young adults, our results also show a very short BTS duration for three of the aged subjects, who completed the BTS in less than 1.1 seconds, which is comparable with the shorter duration of young adults. Interestingly, these subjects also present the smaller BTS trunk angles. These three subjects adopt a different behavior: they drop onto the chair.

Interestingly, the multiple regression shows that BTS trunk angle and STS ratio parameters (strongly linked with trunk flexion) were significantly linked to the functional abilities of all the subjects of both groups. These results strengthened our observation that trunk angle impairments are interesting markers of global changes, since they are highly correlated with functional capacities. Moreover, we have to mention our three worrying subjects presented above, once again highlighted in Figure $2 \mathrm{~B}$ and $\mathrm{C}$. These three subjects presented the worst functional capacities of our frail group, and present both smaller BTS duration and BTS trunk angles. Accordingly, we propose that these parameters are interesting markers of motion disorganization, reflecting impairments in movement planning.

In light of the studies cited above and our results, we propose, even if these speculations need to be verified by further studies, that the BTS motion disorganization is seen with three grades of severity: i) a longer BTS duration due to more cautious motion in normal aging; ii) longer BTS duration accompanied by a smaller trunk angle marking the beginning of a frail state; and iii) the trunk angle vanishing associated with a short BTS duration (dropping on the chair). This last case probably translates a very frail state, potentially associated with cognitive impairments, such as AD.

The system used in this study allowed us to calculate kinematic parameters aimed at revealing small (delicate) changes in motor skills. Indeed, the TUG test has a shortcoming: it is only based on the TUG duration in order to evaluate the overall performance of the task. It therefore lacks specific information on components of each task, such as STS and BTS movements, that could reveal some problems of motion disorganization. Indeed, if the TUG is widely used and well validated for the quantitative aspect (duration in seconds), the different stages of this test require the execution of several complex movements whose quality is not taken into account in the final result of the test. Our results have important clinical implications on geriatric health, especially for the frail population. We found that there are kinematic parameters that reveal delicate changes in fine motor function. These parameters reveal a potential deficiency during the planning processes. We propose that they should be assessed in clinical practice with motion tracking devices, or by observing the trunk motion during transfers. Further studies are needed to 
determine the potential interest of this kinematic assessment in a preventive approach, to allow earlier detection of balance function impairment.

Our study presents several potential limits. First, the number of participants in our study is relatively small (only ten older adults). It could be interesting to obtain a larger sample size to better link kinematic parameters with functional capacities. Moreover, the proportions of males and females are not equal in our effectives. The potential differences among them could represent a bias in our results. To take into account this potential bias, we compared the trunk angles between the two populations: it was not statistically significantly different between males and females ( $P>0.05$ during both STS and BTS).

Second, one may wonder if the kinematic changes we noted are in fact due to the biomechanical constraints linked to aging processes and sedentary behavior of frail patients (stiffness increases, impacting lumbopelvic mobility). However, we emphasize that all the subjects included in the FG were able to move in their houses independently, and to climb up several steps on a staircase. This suggests that the FG subjects have an acceptable hip range of motion.

\section{Conclusion}

Seven kinematic parameters were calculated in order to measure fine changes in motor skills. Significant differences between FG and YG subjects were found in trunk angles and TUG duration. In addition, the BTS trunk angle and STS ratio could allow us to identify motor frailty. These results indicate that there was a relationship between various kinematic changes, representing changes in the motor planning processes, and physical frailty in older people. We propose that these motor planning strategies should be assessed in clinical practice.

\section{Disclosure}

The authors report no conflicts of interest in this work.

\section{References}

1. Chen X, Mao G, Leng SX. Frailty syndrome: an overview. Clin Interv Aging. 2014;9:433-441.

2. Fried LP, Tangen CM, Walston J, et al; Cardiovascular Health Study Collaborative Research Group. Frailty in older adults: evidence for a phenotype. J Gerontol A Biol Sci Med Sci. 2001;56(3):M146-M156.

3. Rastogi R, Meek BD. Management of chronic pain in elderly, frail patients: finding a suitable, personalized method of control. Clin Interv Aging. 2013;8:37-46.

4. Galán-Mercant A, Cuesta-Vargas AI. Differences in trunk accelerometry between frail and non-frail elderly persons in functional tasks. BMC Res Notes. 2014;7:100.

5. Biolo G, Cederholm T, Muscaritoli M. Muscle contractile and metabolic dysfunction is a common feature of sarcopenia of aging and chronic diseases: from sarcopenic obesity to cachexia. Clin Nutr. 2014;33(5):737-748
6. Bonnefoy M. [Sarcopenia, muscle function and prevention]. Nutr Clin Metabol. 2004;18:175-180. French.

7. Woo N, Kim SH. Sarcopenia influences fall-related injuries in communitydwelling older adults. Geriatr Nurs. 2014;35(4):279-282.

8. Gariballa S, Alessa A. Sarcopenia: prevalence and prognostic significance in hospitalized patients. Clin Nutr. 2013;32(5):772-776.

9. Morley JE, von Haehling S, Anker SD, Vellas B. From sarcopenia to frailty: a road less traveled. J Cachexia Sarcopenia Muscle. 2014;5(1):5-8.

10. Saimpont A, Mourey F, Manckoundia P, Pfitzenmeyer P, Pozzo T. Aging affects the mental simulation/planning of the "rising from the floor” sequence. Arch Gerontol Geriatr. 2010;51(3):e41-e45.

11. Allain P, Chaudet H, Nicoleau S, et al. Étude de la planification de l'action au moyen du test du plan du zoo dans la maladie d'Alzheimer. [A study of action planning in patients with Alzheimer's disease using the zoo map test]. Rev Neurol (Paris). 2007;163(2):222-230. French.

12. Mourey F, Pozzo T, Rouhier-Marcer I, Didier JP. A kinematic comparison between elderly and young subjects standing up from and sitting down in a chair. Age Ageing. 1998;27(2):137-146.

13. Karthikbabu S, Solomon JM, Manikandan N, Rao BK, Chakrapani M, Nayak A. Role of trunk rehabilitation on trunk control, balance and gait in patients with chronic stroke: a pre-post design. Neurosci Med. 2011;2(2):61-67.

14. Massion J. Movement, posture and equilibrium: interaction and coordination. Prog Neurobiol. 1992;38(1):35-56.

15. Manckoundia P, Mourey F, Pfitzenmeyer P, Papaxanthis C. Comparison of motor strategies in sit-to-stand and back-to-sit motions between healthy and Alzheimer's disease elderly subjects. Neuroscience. 2006;137(2):385-392.

16. Buchman AS, Boyle PA, Wilson RS, Tang Y, Bennett DA. Frailty is associated with incident Alzheimer's disease and cognitive decline in the elderly. Psychosom Med. 2007;69(5):483-489.

17. Buchman AS, Schneider JA, Leurgans S, Bennett DA. Physical frailty in older persons is associated with Alzheimer disease pathology. Neurology. 2008;71(7):499-504.

18. Solfrizzi V, Scafato E, Frisardi V, et al; Italian Longitudinal Study on Aging Working Group. Frailty syndrome and the risk of vascular dementia: the Italian Longitudinal Study on Aging. Alzheimers Dement. 2013;9(2):113-122.

19. Podsiadlo D, Richardson S. The timed "Up and Go": a test of basic functional mobility for frail elderly persons. J Am Geriatr Soc. 1991; 39(2):142-148.

20. McGrath D, Greene BR, Doheny EP, McKeown DJ, De Vito G, Caulfield B. Reliability of quantitative TUG measures of mobility for use in falls risk assessment. Conf Proc IEEE Eng Med Biol Soc. 2011;2011:466-469.

21. Whitney SL, Marchetti GF, Schade A, Wrisley DM. The sensitivity and specificity of the Timed "Up and Go" and the Dynamic Gait Index for self-reported falls in persons with vestibular disorders. J Vestib Res. 2004;14(5):397-409.

22. Shumway-Cook A, Brauer S, Woollacott M. Predicting the probability for falls in community-dwelling older adults using the Timed Up and Go Test. Phys Ther. 2000;80(9):896-903.

23. Bonnechère $B$, Jansen $B$, Salvia $P$, et al. What are the current limits of the Kinect ${ }^{\mathrm{TM}}$ sensor? In: Ninth International Conference on Disability, Virtual Reality and Associated Technologies (ICDVRAT); September 10-12, 2012; Laval, France: 287-294.

24. Hassani A, Kubicki A, Brost V, Yang F. Preliminary study on the design of a low-cost movement analysis system: reliability measurement of Timed Up and Go test. In: The Ninth International Conference on Computer Vision Theory and Applications; January 5-8, 2014; Lisbon, Portugal: 33-38.

25. Thalheimer W, Cook S. How to Calculate Effect Sizes from Published Research: A Simplified Methodology. Somerville, MA; Work-Learning Research; 2002.

26. Cignetti F, Chabeauti PY, Sveistrup H, Vaugoyeau M, Assaiante C. Updating process of internal models of action as assessed from motor and postural strategies in children. Neuroscience. 2013;233:127-138. 
27. Papaxanthis C, Dubost V, Pozzo T. Similar planning strategies for whole-body and arm movements performed in the sagittal plane. Neuroscience. 2003;117(4):779-783.

28. Man'kovskii NB, Mints AYa, Lysenyuk VP. Regulation of the preparatory period for complex voluntary movement in old and extreme old age. Hum Physiol. 1980;6(1):46-50.
29. Woollacott MH, Manchester DL. Anticipatory postural adjustments in older adults: are changes in response characteristics due to changes in strategy? J Gerontol. 1993;48(2):M64-M70.

30. Kubicki A, Bonnetblanc F, Petrement G, Ballay Y, Mourey F. Delayed postural control during self-generated perturbations in the frail older adults. Clin Interv Aging. 2012;7:65-75.

\section{Publish your work in this journal}

Clinical Interventions in Aging is an international, peer-reviewed journal focusing on evidence-based reports on the value or lack thereof of treatments intended to prevent or delay the onset of maladaptive correlates of aging in human beings. This journal is indexed on PubMed Central, MedLine,

\section{Dovepress}

CAS, Scopus and the Elsevier Bibliographic databases. The manuscript management system is completely online and includes a very quick and fair peer-review system, which is all easy to use. Visit http://www.dovepress. $\mathrm{com} /$ testimonials.php to read real quotes from published authors. 